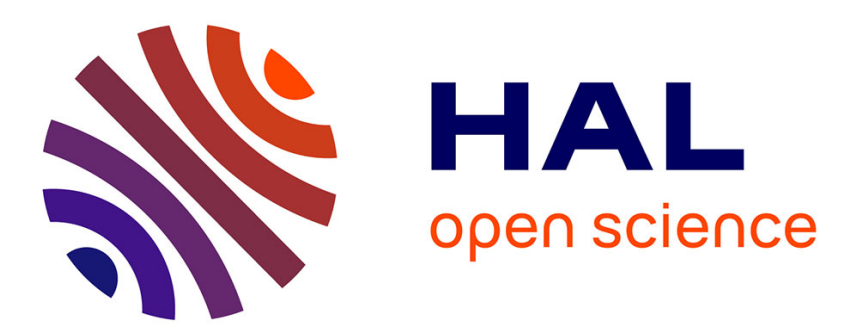

\title{
Faire face à l'échec: stratégies d'évitement, adaptabilité et modernisation dans le commerce des vins languedociens (1900-1950)
}

Stéphane Le Bras

\section{- To cite this version:}

Stéphane Le Bras. Faire face à l'échec: stratégies d'évitement, adaptabilité et modernisation dans le commerce des vins languedociens (1900-1950). L'échec a-t-il des vertus économiques?, Comité pour l'histoire économique et financière de la France / IGPDE, pp.275-289, 2015, 978-2-11-129397-7. hal-01325324

\section{HAL Id: hal-01325324 \\ https://hal.science/hal-01325324}

Submitted on 2 Jun 2016

HAL is a multi-disciplinary open access archive for the deposit and dissemination of scientific research documents, whether they are published or not. The documents may come from teaching and research institutions in France or abroad, or from public or private research centers.
L'archive ouverte pluridisciplinaire HAL, est destinée au dépôt et à la diffusion de documents scientifiques de niveau recherche, publiés ou non, émanant des établissements d'enseignement et de recherche français ou étrangers, des laboratoires publics ou privés. 


\title{
FAIRE FACE À L'ÉCHEC : STRATÉGIES D'ÉVITEMENT, ADAPTABILITÉ ET MODERNISATION DANS LE COMMERCE DES VINS LANGUEDOCIENS
}

(1900-1950)

\author{
Stéphane Le Bras \\ Univ. Blaise-Pascal, CHEC
}

La question de l'échec est au cœur des problématiques commerciales qui structurent le métier de négociant en vins en Languedoc. Interface entre le monde de la propriété dont il doit écouler la production $^{1}$ et celui de la consommation dont il doit répondre aux besoins, la maison de commerce est soumise à de nombreuses tensions qui fragilisent son activité et, plus ou moins rapidement, concourent à son échec, ponctuel ou permanant.

Cette thématique recoupe trois domaines fondamentaux dans la profession. La première est la question de la réputation. C'est là un élément essentiel à la bonne marche des maisons de commerce qui investissent de nombreuses stratégies pour consolider leur réputation ou, au contraire, l'amender. La seconde est la grande vulnérabilité de cette profession. Celle-ci est soumise à de multiples risques qui font peser sur les épaules du négociant une forte pression, source et conséquence d'échecs : source car elle le pousse à prendre des risques ; conséquence car les échecs qui se multiplient autour de lui soulignent cette menace constante. La dernière enfin est l'instabilité de la conjoncture sur le marché des vins languedociens. Doublée d'une croissance marquée de la concurrence, la période est caractérisée par des périodes de profits vertigineux suivies de dépressions tout aussi rapides².

Dans ce contexte, le négociant à la tête d'une maison de commerce doit mettre en œuvre des stratégies qui lui permettent de faire face à l'échec, élément consubstantiel du marché des vins languedocien, lequel est particulièrement conditionné par l'incertitude 3 .

Pour présenter ces stratégies déterminées par le désir d'éviter les échecs, nous verrons quels sont les différents risques de déboires sur le marché des vins (I), puis les multiples moyens déployés pour préserver sa réputation et éviter les revers (II).

\footnotetext{
${ }^{1}$ Le Languedoc (Aude, Gard et Hérault) est la plus grande région productrice de France pour la période étudiée durant laquelle elle fournit entre 30 et $40 \%$ de la production nationale. Pour des chiffres détaillés, voir Marcel Lachiver, Vins, vignes et vignerons. Histoire du vignoble français, Paris, Fayard, 1988, 714 p.

2 Pour une plus large contextualisation voir Geneviève Gavignaud-Fontaine, Le Languedoc viticole, la Méditerranée et l'Europe au siècle dernier (XX $X^{e}$ s.), Montpellier, Presses de l'Université Paul-Valéry, 2006, 528 p.

${ }^{3}$ Bien qu'on soit ici très loin du marché des singularités étudiés par Lucien Karpik, on y retrouve cette dominante forte : la nécessaire gestion de l'incertitude. Cf. Lucien Karpik, L'économie des singularités, Paris, Gallimard, 2007, 373 p.
} 


\section{DES ÉCHECS PROTÉIFORMES SUR LE MARCHÉ DES VINS LANGUEDOCIENS}

\section{A. LA SPÉCULATION, FACTEUR D’ÉCHEC}

Le négociant, par sa fonction sur le marché des vins, est un spéculateur. Il passe avec ses partenaires commerciaux des contrats qui requièrent une parfaite connaissance du marché et un sens développé pour l'anticipation et la prise de risque. Ses stratégies d'achat, lorsqu'il achète au forfait ${ }^{4}$, sont de deux types : à la hausse ou à la baisse. Dans le premier cas, le négociant haussier est confiant dans la dynamique ascensionnelle du marché. Il achète donc tôt dans la campagne (septembre-octobre) ou avant les fêtes (fin novembre ou mars) des quantités souvent importantes à un prix assez bas afin de les revendre quelques temps plus tard à des cours plus élevés. Il cherche ainsi à devancer les demandes de ses concurrents et de ses clients dans les semaines et les mois à venir. Dans le second cas, le négociant baissier cherche à vendre son vin avant de l'avoir acheté. Tablant sur une baisse des cours, il passe un contrat avec un client à un tarif donné en espérant que dans les semaines à venir, entre le moment du contrat et de l'expédition, les cours auront chuté. C'est ce qu'on appelle la « vente à découvert $»^{5}$ et c'est une stratégie spéculative qui vise à attirer la clientèle par une gamme de prix attractive, inférieure à celle des concurrents.

Cette recherche de bénéfices par le biais de la spéculation est parfaitement admise dans le monde du commerce en général ${ }^{6}$ et dans la société viti-vinicole en particulier ${ }^{7}$ au cours du premier $\mathrm{XX}^{\mathrm{e}}$ siècle. Mais si elle est source de profits remarquables, elle est également à l'origine d'échecs commerciaux parfois retentissants. Certains haussiers, par exemple, pris par la chute des cours, sont obligés de « vendre à crédit », c'est-à-dire à perte. C'est le cas par exemple de la maison « Castelbon » à Béziers en $1904^{8}$ ou d'Auguste Taillan au début des années 1910 à Sète ${ }^{9}$. Certaines de ces pratiques spéculatives trop risquées entraînent même la disparition de maisons de commerce solidement établies, comme « Leenhardt-Bazille » à Montpellier dans la décennie suivante ${ }^{10}$. Une des maisons les plus puissantes et les plus anciennes de la place, «Leenhardt-Bazille» fait faillite après que la direction eut été confiée à un héritier inconsistant et à des directeurs commerciaux trop «ardents » selon la terminologie des rapports d'inspection de l'époque. La famille est alors obligée de se séparer de certains de ses biens et de faire appel à un « conseil familial » pour gérer la liquidation d'une des

\footnotetext{
${ }^{4}$ Le négociant forfaitaire est un négociant qui dispose de chais et achète en grande quantité des vins qu'il stocke, entretient et coupe selon les désirs de la clientèle. C'est la catégorie dominante sur le marché des vins jusqu'à l'entre-deux-guerres. L'autre type de négoce est le négoce à la commission qui, lui, ne fait que relayer les ordres d'un commettant.

${ }^{5}$ Pierre Bergé, Le marché des vins du Midi, Paris, PUF, 1927, p. 34.

${ }^{6}$ Voir à ce sujet Alessandro Stanziani, «Les deux sens de la spéculation au XIX ${ }^{\mathrm{e}}$ siècle » in Alya Aglan, Olivier Feiertag, Yves Marec, Les Français et l'argent, entre fantasmes et réalités, Rennes, PUR, 2011, p. 267-279.

${ }^{7}$ L'activité de spéculation est perçue comme une pratique régulatrice sur le marché des vins et même les plus farouches adversaires du négoce la justifient. Ainsi, Paul Degrully, partisan de la vente directe, en reconnaît à de multiples reprises l'utilité dans Paul Degrully., Essai historique et économique sur la production et le marché des vins en France, Montpellier, Goulet et fils, 1910, 466 p.

${ }^{8}$ Archives de la Banque de France [désormais Arch. BdF] : rapport d'inspection de la succursale de Béziers [désormais : Béziers], 1905.

${ }^{9}$ Arch. BdF : Sète, 1912.

${ }^{10}$ Arch. BdF : Montpellier, 1922.
} 
plus anciennes maisons de la place. Bien que moins risquée, la spéculation à la baisse peut également entraîner des échecs. Ainsi, en 1912, deux vendeurs à découvert de la région sont forcés de trouver un arrangement avec leur client. Alors qu'ils avaient prévu de vendre $90.000 \mathrm{hl}$ à la baisse, la hausse subite et brusque des cours risque de leur faire perdre $2 \mathrm{M}$ de F. Ils s'entendent alors devant la justice à verser $1 \mathrm{~F}$ par hl de dommages et intérêts à leur client, ce qui représente une perte moins substantielle ${ }^{11}$.

Si la faillite n'est pas toujours la conséquence de ces échecs, ces derniers fragilisent les maisons de commerce sur les plans financiers et réputationnels. C'est également le cas lors des poursuites pour fraudes.

\section{B. ÉCHEC À LA FRAUDE ET ÉCHECS DES FRAUDEURS}

La fraude est également un élément constitutif et indissociable du marché des vins languedociens. Elle est présente partout et prend plusieurs formes. On la retrouve au cœur du discours des organisations patronales (le Syndicat régional du Commerce en gros des vins et spiritueux du Midi par exemple), des corps consultatifs (chambres de commerce) ou des hommes politiques. Ses formes sont multiples : fraude fiscale ${ }^{12}$, fraude commerciale ${ }^{13}$, fraude sur la marchandise ${ }^{14}$. La fraude représente un échec lorsque le négociant est poursuivi et, dès lors, elle a une double conséquence : financière lorsqu'elle est assortie d'une peine ou d'une amende ; réputationnelle dès l'entame des poursuites.

Les rapports d'inspection de la Banque de France $(\mathrm{BdF})$ et la presse spécialisée recèlent de cas de fraudes - volontaires ou non - qui ternissent l'image des maisons de commerce incriminées. En 1903, l'inspecteur de la BdF relève à propos de la maison «Combier et Cie » : «Fraudeurs, toujours sous le coup de poursuites de la part de la Régie. Être prudent et s'abstenir. ${ }^{15}$ Ainsi, la banque se montre méfiante quant au crédit à accorder à une maison dont la réputation a été écornée par une pratique généralisée dans la région biterroise ${ }^{16}$ et au-delà ${ }^{17}$. Dans le même ordre d'idées, en 1931, les frères d'une maison réputée de Frontignan sont condamnés à un an de prison pour fabrication de faux Porto $^{18}$. Enfin, au début des années 1950, un rapport du Service de la répression des fraudes ${ }^{19}$ indique que les fraudes sont encore nombreuses sur le marché des vins et qu'elles n'ont pas disparu en dépit

\footnotetext{
${ }^{11}$ Moniteur Judiciaire du Midi [désormais MJM], 07/07/1912, Cour d'Appel de Nîmes (séance du 19/02/1912).

12 Fraudes sur les entrées et les sorties des magasins, trafics de documents administratifs et de circulation.

${ }^{13}$ Fraudes publicitaires

14 Vins impropres à la consommation, vins mouillés, vins ne correspondant pas aux qualités et quantités prévues par le contrat.

15 Arch. BdF: Béziers, 1903.

16 Arch. BdF: Béziers, 1902.

${ }^{17}$ Le Progrès vinicole dans son édition du 05 avril 1900 évoque les « Baptistères » à Paris, « usines [à vin] dans lesquelles quelles le vin entre à 14 ou 15 degrés et n'est ensuite livré aux clients des tenanciers de ces fabriques qu'aux environs de 10 degrés ».

18 Arch. BdF: Sète, 1931.

${ }^{19}$ Arch. nat. de Fontainebleau : 19800139/35, Rapport annuel du Service de répression des fraudes, 1951.
} 
des mouvements de protestations populaires (1907 par exemple ${ }^{20}$, doc. 1) ou des lois encadrant le marché (Statut viticole voté dans les années 1930²1).

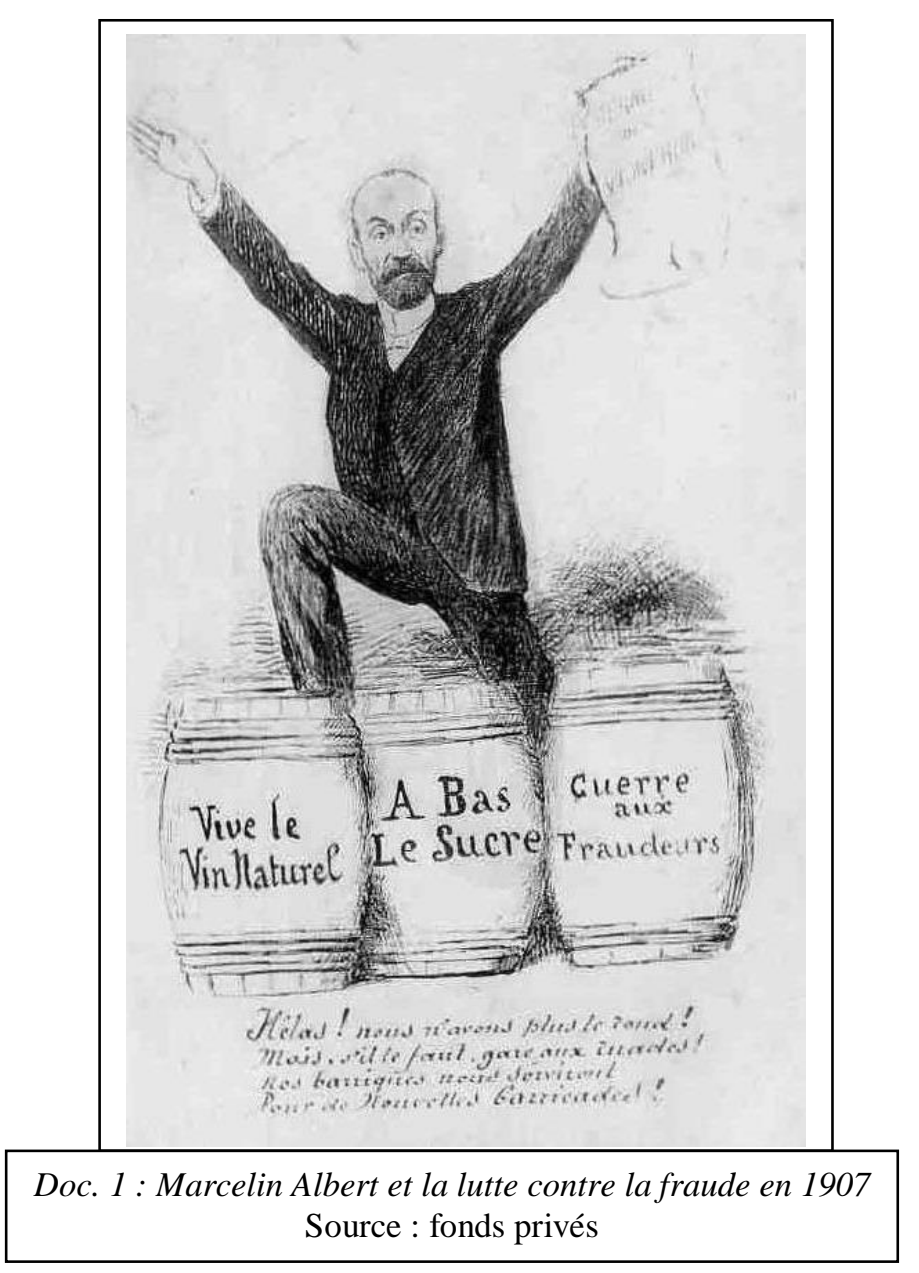

En creux, on lit dans ce constat l'échec des syndicats des vins et de l'État sur le marché des vins. Les premiers, dans une double dynamique de recherche d'assainissement du marché et de consolidation de la respectabilité de la profession, se sont lancés depuis le début du siècle dans une démarche lobbyiste visant à limiter les impacts dépréciatifs des fraudes. Le second, par son désir de réguler un marché toujours difficilement contrôlable et sur lequel les pratiques frauduleuses sont nombreuses, a cherché à mettre en place des structures de contrôle plus ou moins efficaces (législation comminatoire (1889, 1905, 1907 par exemple ${ }^{22}$ ), Service de répression des fraudes, encadrement des cours). Indéniablement, c'est là un type d'échec - institutionnel - qui dépasse le cadre des maisons de commerce mais est révélateur des difficultés et des tensions sur le marché des vins.

C'est pour cette raison que les négociants sont particulièrement attentifs, dans ce contexte préoccupant et tendu, à s'adapter aux conditions du marché.

\footnotetext{
${ }^{20}$ Pour de plus amples précisions voir Jean Sagnes (dir.), La révolte du Midi viticole : cent ans après, 1907-2007, Presses universitaires de Perpignan, Perpignan, 2008, 345 p.

${ }^{21}$ À ce sujet, voir Jean-Marc Bagnol, Le Midi viticole au Parlement. Édouard Barthe et les députés du vin de l'Hérault (années 1920-1930), Montpellier, Presses Universitaires de la Méditerranée, 2011, 462 p.

22 Alessandro Stanziani, «La falsification du vin en France, 1880-1905: un cas de fraude agro-alimentaire », Revue d'histoire moderne et contemporaine, 2003-2, p. 154-186.
} 


\section{LES VERTUS DE L'ÉCHEC : L’ADAPTABILITÉ DES MAISONS DE COMMERCE}

\section{A. LE CONTRÔLE DE L'INFORMEL}

Dans un monde viti-vinicole où la question de l'informel est primordiale, son contrôle assure, dans une certaine mesure, la limitation des risques et des échecs.

Dans un premier temps, la maîtrise des informations est essentielle. Pour ce faire, les négociants se rendent régulièrement sur les marchés aux vins qui jalonnent la semaine dans le $\mathrm{Midi}^{23}$ : Lundi à Perpignan et Nîmes; Mardi à Montpellier et Carcassonne; Mercredi à Sète ; Jeudi à Narbonne; Vendredi à Béziers ; Samedi à Pézenas. Le passage par le marché est crucial pour connaître les grandes tendances et pouvoir acheter au «juste prix» ou adapter ses stratégies commerciales. Il faut être à l'écouter des rumeurs, toujours importantes, pour prévoir la conjoncture. On y rencontre des connaissances qui font état de bonnes affaires à réaliser ou de caves à éviter (doc. 2).

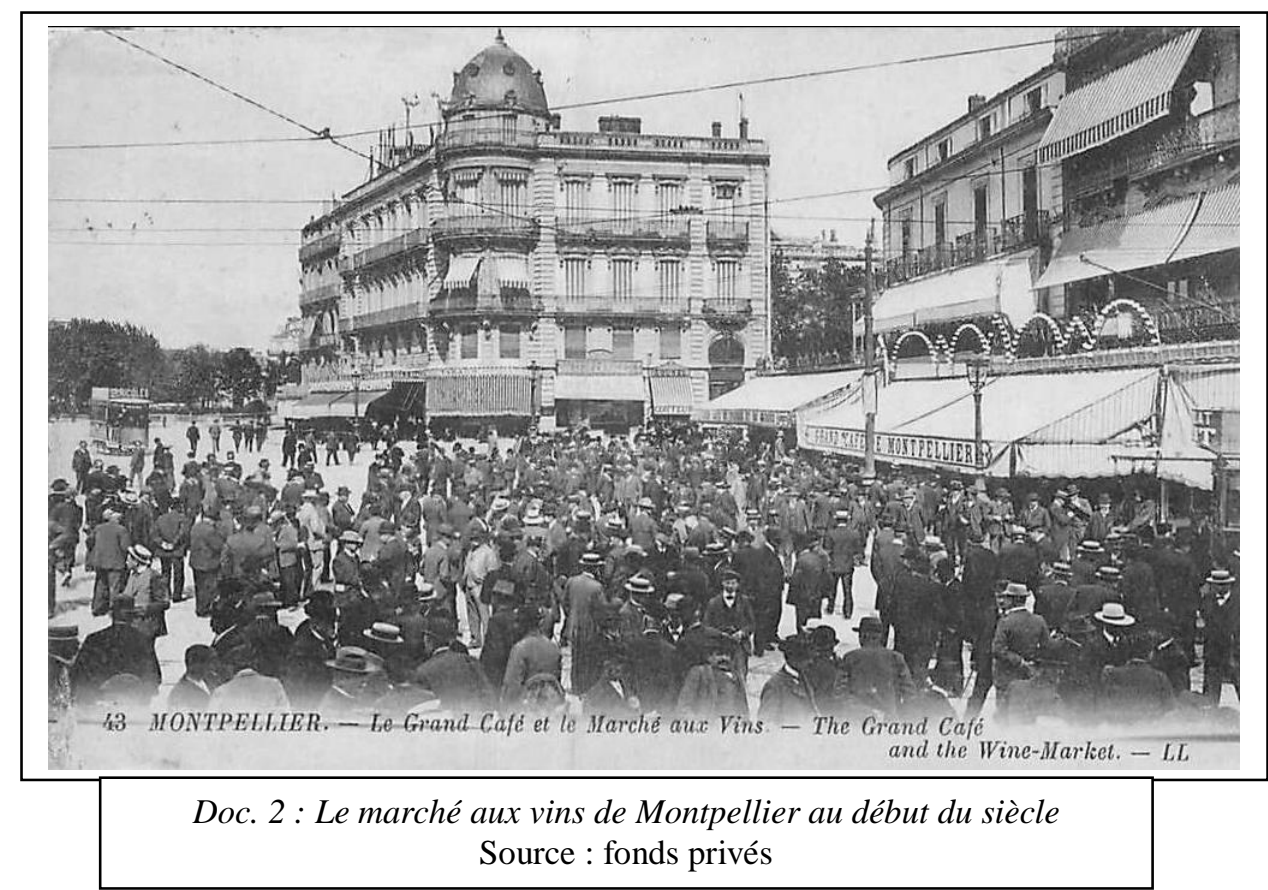

Ce contrôle de l'information mercuriale est l'enjeu de nombreuses tensions et rivalités entre partenaires commerciaux. Les clients réclament à certains de leurs fournisseurs des comptes rendus précis de l'état du marché tandis que les journaux n'hésitent pas à propager de fausses rumeurs selon leur orientation (proximité avec le négoce ou proximité avec les propriétaires). Ainsi en 1911, le Midi Vinicole répond à certains de ses lecteurs qui l'accusent de propager de fausses rumeurs et d'être baissier ${ }^{24}$. Quelques années plus tard, le Petit Méridional évoque «les fausses rumeurs adroitement

\footnotetext{
${ }^{23}$ Léopold Vivarès, «Les grands marchés vinicoles du Midi », Bulletin de la Société languedocienne de géographie, Tome XLI, 1918, p. 22-36.

${ }^{24}$ Midi Vinicole, 20/06/1911.
} 
colportées les jours de marché aux vins [qui] jettent le discrédit et le découragement parmi les commerçants et viticulteurs honnêtes $»^{25}$. Dans le domaine commercial, ce contrôle est également important. Les négociants n'hésitent pas à écrire aux banques ${ }^{26}$ ou à faire appel à des sociétés spécialisées ${ }^{27}$ (doc. 3) qui enquêtent sur des clients potentiels (crédit, respectabilité, type de commerce, longévité, poursuites, etc.). C'est là une stratégie vitale pour se protéger et éviter les risques d'échec et de déboire commerciaux.

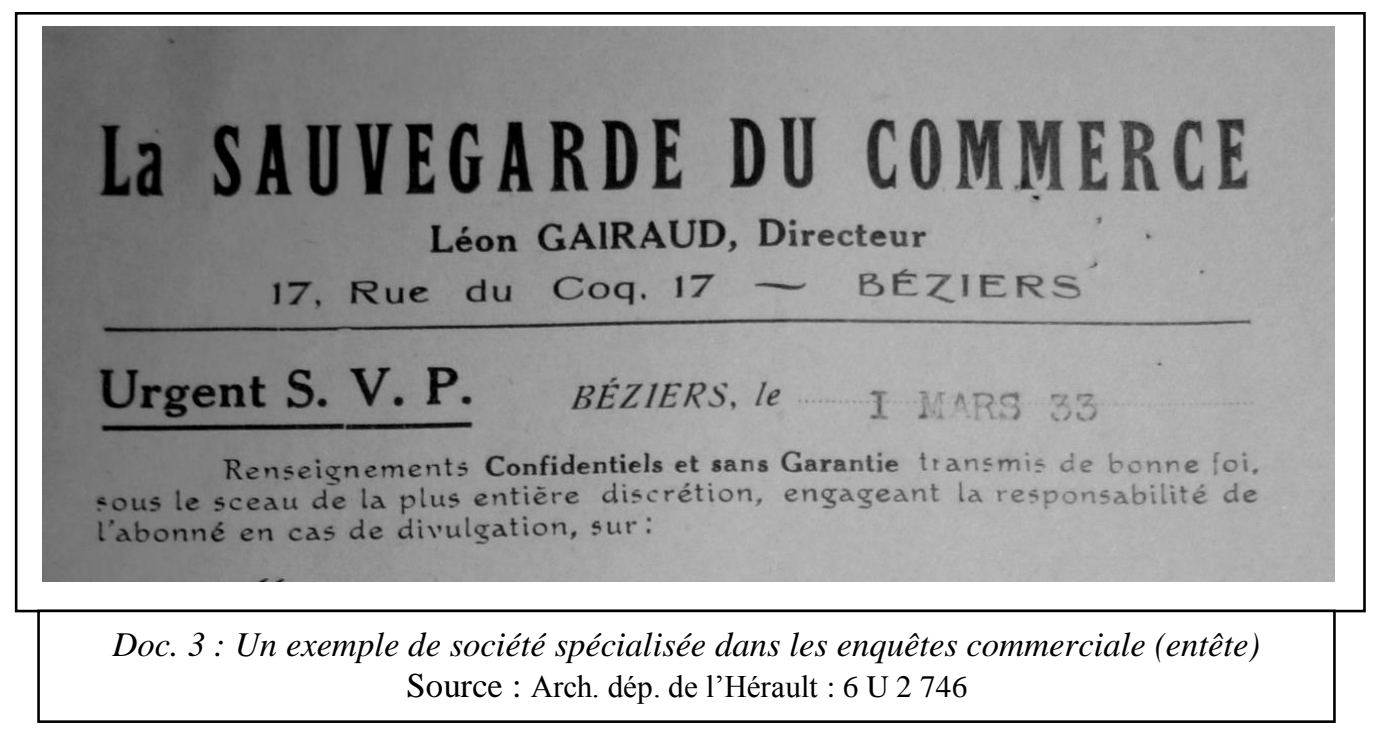

\section{B. LA MUTATION STRUCTURELLE}

Le second type de stratégies visant à éviter les échecs est la mutation commerciale. Celle-ci peut prendre plusieurs formes. Dans un contexte de crises à répétitions et de multiplication des risques, les patrons choisissent des pratiques d'achats les moins risquées. Tout d'abord, les achats à la baisse se généralisent ${ }^{28}$ : ils deviennent majoritaires en raison des faibles besoins en capitaux qu'ils nécessitent, mais surtout car ils limitent les risques de perte. Ensuite, et surtout, les ventes à la commission prennent de plus en plus le pas sur les ventes au forfait. Si les commissionnaires purs ${ }^{29}$ sont encore peu nombreux au début du siècle, leur nombre augmente rapidement au cours des décennies 1910, 1920 et 1930. À Béziers, s'ils représentent moins de $10 \%$ des maisons en 1902, ils en représentent plus de la moitié à partir de 1920. Dans les décennies suivantes, leur part tourne autour de $50 \%$, avec des fluctuations selon la conjoncture : à Béziers où le tissu commercial est plus fragile que dans les autres grands centres d'expédition héraultais, plus les temps sont difficiles, plus le nombre de

\footnotetext{
${ }^{25}$ Petit Méridional, 30/07/1920.

${ }^{26}$ Arch. dép. de l'Hérault : 5 J 35, Fonds A. Domergue, négociant en vin à Montpellier, Correspondance, 1906. Il écrit à la banque « Tissié-Sarrus » à propos de maisons faisant partie de la clientèle de la banque montpelliéraine.

27 Arch. dép. de l'Hérault : 6 U 2 746, Faillite Augé, négociant en vins à Cessenon, correspondance, 1929. Augé fait régulièrement appel à la société «La Sauvegarde du Commerce » qui enquête sur les maisons de commerce extraméridionales.

${ }^{28}$ P. Bergé, Les marchés..., op. cit., p. 35.

${ }^{29} \mathrm{Ce}$ sont les maisons qui ne pratiquent que la vente à la commission.
} 
commissionnaires augmentent ${ }^{30}$. Le recours à ce type de structures, surtout lorsqu'on a connu la faillite, est logique. Il requiert moins de fonds, est moins taxé (jusqu'en 1930) et limite les responsabilités dans la transaction. Dès le début du siècle et les crises de mévente, la quasi-totalité des maisons de commerce spécialisées dans les VCC écoulent une partie de leur marchandise par ce biaislà.

Dans certains cas, certains faillis restent dans la filière, mais avec d'autres occupations ou d'autres noms. Ainsi, Raphaël Tous qui avait hérité d'une des plus anciennes maisons sétoises («B. Tous ») et qui par son mariage avait absorbé une autre vieille maison de la ville («P. Hubidos») fait faillite au milieu des années 1930 après une opération malheureuse ${ }^{31}$. À la fin des années 1930, on le trouve référencé comme «courtier» dans l'Annuaire de l'Hérault ${ }^{32}$ et après la Seconde Guerre mondiale, il devient directeur des opérations commerciales chez « Cazalis \& Prats », l'une des plus grandes maisons sétoises. D'autres négociants faillis ou fraudeurs n'hésitent pas à continuer leur commerce par le biais de prête-noms. C'est le cas par exemple du mézois Allègre au début du siècle «fraudeur, dont la moralité laisse à désirer » qui cède sa société à ses filles ${ }^{33}$. À Bédarieux, dans les années 1930, Joseph Brun, «mal réputé », a été mis en liquidation judiciaire mais il continue le commerce sous le nom de sa femme, Marie $^{34}$ (doc. 4).

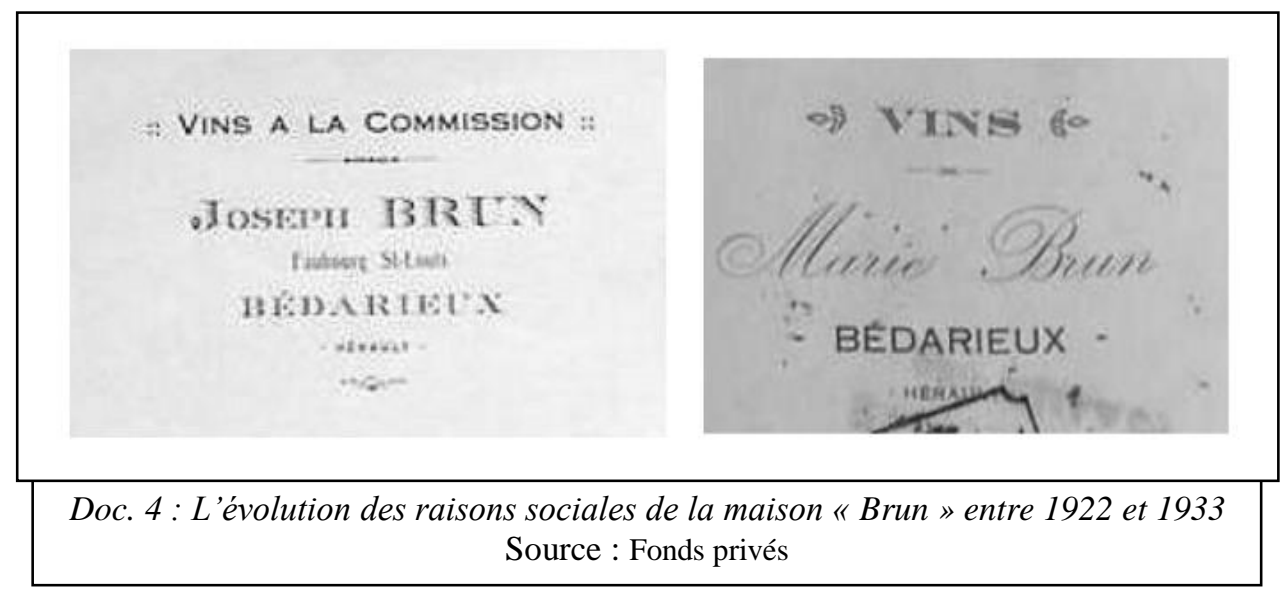

Cela explique que, d'un sondage à l'autre, le roulement des raisons sociales soit important ${ }^{35}$, mais qu'en réalité il ne concerne qu'un nombre limité de négociants.

Enfin, pour réduire les risques et gagner en efficacité, les structures des maisons de commerce se transforment également. Les années 1910 voient la timide émergence des sociétés anonymes (SA) qui se multiplient dans les années 1920 et 1930. Surtout, à partir de la loi de 1926, la plupart des maisons de commerce qui étaient jusque-là gérées sous le régime des sociétés en nom collectif ou des sociétés en commandite simple, passent sous le statut des sociétés à responsabilité limitée (SARL). SA et SARL permettent de mieux gérer les fonds et les capitaux, mais surtout limitent les risques en cas

\footnotetext{
${ }^{30}$ Annuaire de l'Hérault, 1900-1939.

${ }^{31}$ Arch. BdF : Montpellier, 1934 et Sète, 1935. Il a acheté des vins sur souche (c'est-à-dire avant leur récolte) à un prix deux fois plus élevé que les cours.

${ }^{32}$ Annuaire de l'Hérault, 1939.

${ }^{33}$ Arch. BdF : Sète, 1882 et 1901

${ }^{34}$ Arch. BdF : Béziers, 1933.

$3539 \%$ de nouvelles maisons entre 1900 et 1919 et $47 \%$ entre 1920 et 1929 à Sète.
} 
d'échecs commerciaux, faisant peser la responsabilité sur les structures et non plus les individus. Ainsi, la grande majorité des maisons languedociennes («Byrrh», «Cazalis \& Prats », «P. Taillan », «V. Anthérieu», «Bartissol») prennent cette forme, imitant en cela les maisons extra-méridionales (« Dubonnet», «Société des vins de France », « Noilly-Prat», «Vins du Postillon», « Nicolas », etc.)

Dans le même temps, les maisons modernisent leurs techniques commerciales.

\section{De NOUVELLES TEChNiQues DE VENTE ET DE STOCKAGE}

La crainte de l'échec a enfin comme vertu la nécessaire modernisation d'une profession jugée au début du siècle comme immobiliste et artisanale. Cette modernisation se fait dans trois directions.

La première est le recours massif à la publicité. Cette dernière devient un élément stratégique de premier ordre dans les modèles de développement des maisons de commerce et elle prend plusieurs formes. Ici encore, on imite souvent ce que font les maisons à dimension nationale telles Dubonnet. À Marseillan, la maison «J. Voisin» investit massivement dans la publicité. Son patron, Jean Voisin, l'une des fortunes les plus importantes de la ville grâce à son « apéritif tonique Mignon », invente un personnage, «Mademoiselle Mignon», qui vante les produits de la maison. On la retrouve sur des cartes postales, des buvards, des calendriers et sur les prix-courants envoyés aux clients. Ces publicités reprennent les visuels habituels de la maison (Mademoiselle Mignon, port de Marseillan, bouteille) ainsi que les nombreuses récompenses de la maison. Des portes-timbres sont également créés à l'effigie de Mademoiselle Mignon, sur lesquels on trouve la chanson qui l'accompagne partout : « On m'appelle Mignon, je n'ai pas d'autres noms $»^{36}$. Au début du siècle, son fils Urbain Voisin fait appel à un des dessinateurs les plus en vogue de la période, Moriss, pour rénover son image publicitaire (doc. 5).

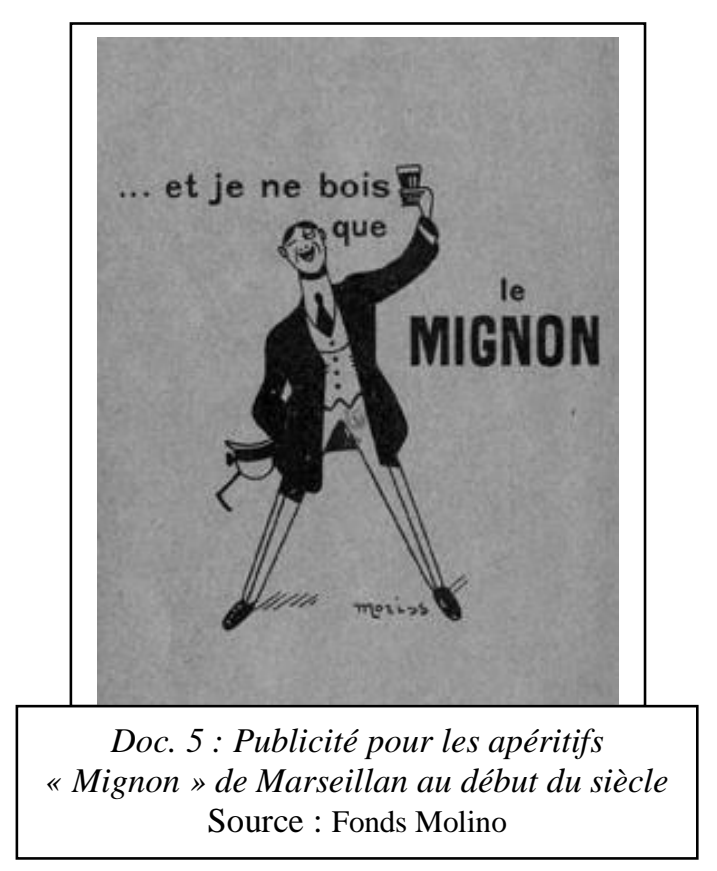

\footnotetext{
${ }^{36}$ Jean-Vincent Molino, Marseillan : de la vigne à la table, Saint-Avertin, Alan Sutton Eds, 2006, p. 68-71.
} 
Depuis Thuir dans les Pyrénées-Orientales, les frères Violet font la publicité de leur quinquina Byrrh que l'on retrouve sur les murs de toutes les communes françaises. Selon la puissance financière des maisons ${ }^{37}$, ces stratégies publicitaires sont investies à différents degrés par les maisons de commerce. Mais depuis les en-têtes stylisées des lettres jusqu'à la publicité radiophonique de la maison « Saint-Raphaël », toutes les maisons s'investissent dans le développement publicitaire.

Dans les chais, cette modernisation est également remarquable. Ceux-ci se modernisent avec de nouveaux outils. Dès le début du siècle, les cuves en ciment remplacent peu à peu les foudres en bois. Plus économiques, plus hygiéniques et plus solides, elles permettent une meilleure conservation des vins. Durant l'entre-deux-guerres, les cuves en ciment se généralisent. Elles sont verrées pour permettre une meilleure fermentation grâce à leur température constante et éviter l'altération des vins par les parois ${ }^{38}$. Par ailleurs d'une contenance supérieure, les cuves en ciment permettent d'accroître les capacités de stockage. À Sète, la maison « Cazalis et Prats » voient leur capacité de stockage passer de $25.000 \mathrm{hl}$ à $230.00 \mathrm{hl}$ sur la période ${ }^{39}$. Les maisons communiquent abondamment dans leur publicité sur ce thème, signe d'une modernité limitant les risques (doc. 6).

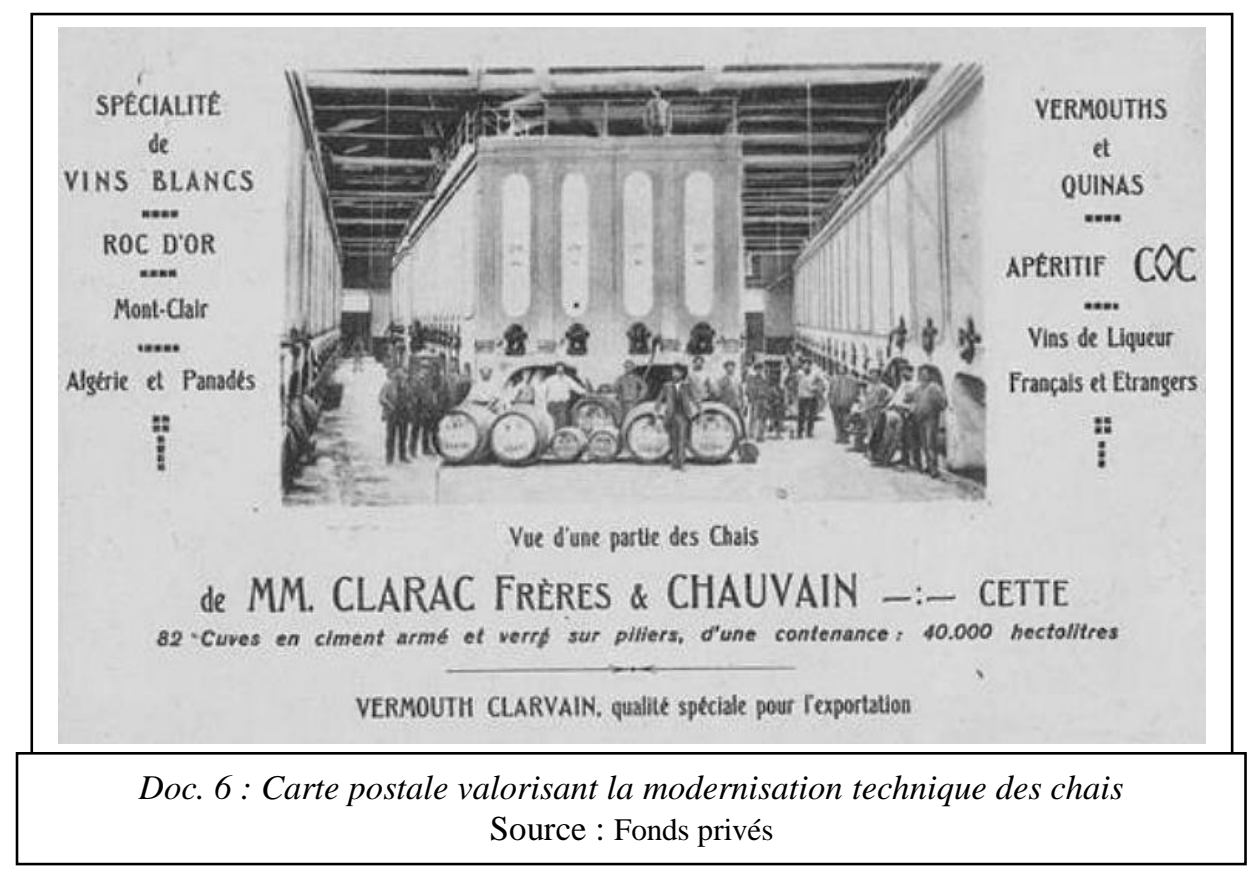

Durant cette même période, l'électricité fait son apparition dans les chais permettant d'utiliser de nouveaux outils de plus en plus performants. Le tableau Daubron tout d'abord qui, dans les années 1920, permet de commander depuis un tableau central relié à de puissantes moto-pompes l'ensemble des mouvements de marchandises dans les chais. À l'aide de vannes (qui dans les années 1940 deviennent des boutons), quelques hommes seulement peuvent effectuer les transvasements depuis les containers jusqu'aux cuves et inversement. Enfin, les pompes elles-mêmes se modernisent: les

\footnotetext{
${ }^{37}$ Devant la Commission Barthe venue enquêter sur les difficultés sur le marché des vins en 1930, un des représentants des négociants reconnaît que cet effort publicitaire est très coûteux. Cf. Édouard Barthe, Rapport fait au nom de la Commission des boisson chargée de procéder à une enquête sur la situation de la viticulture de France et d4algérie et sur toutes les réformes utiles de nature à améliorer le marché vinicole et à réduire les charges qui frappent le commerce des vins, Tome Premier (Hérault, Aude, Pyrénées), Rapport n³156, Impr. de la Chambre des députés, 1932, p. 50.

38 «La fermentation en cuves verrées », Bulletin mensuel du Syndicat des vins montpelliérain, mars-avril 1914, p. 98-99

${ }^{39}$ Publicités diverses.
} 
pompes manuelles, apparues au tournant du siècle, sont remplacées par des pompes mécaniques, à essence ou, dès l'entre-deux-guerres, à électricité. Dans le même temps, les filtres se perfectionnent également, permettant un filtrage plus rapide et plus sûr ${ }^{40}$. Tout est fait pour éviter de dénaturer ou abimer la marchandise, ce qui serait un risque considérable pour des maisons souvent fragilisées par un contexte instable.

$* * *$

La question de l'échec est récurrente dans le commerce des vins languedociens et elle est en permanence à l'esprit des patrons des maisons de négoce, quelle que soit leur taille et quelle que soit l'époque.

Ces échecs sont de natures multiples et peuvent prendre différentes formes mais ils ont tous comme conséquence la fragilisation d'un tissu économique déjà rendu précaire par les crises multiples qui le structurent. Pour faire face à ces échecs, les négociants s'investissent - soit préventivement soit en réaction - dans des stratégies multiples elles-aussi : modernisation, contrôle de l'informel, changement de structures. Si ces pratiques ne sont pas toujours motivées par des principes honorables, elles participent toujours à la stabilisation de la profession et de ses acteurs.

On peut ainsi, à l'aune de cette étude, reconnaître que sur le marché des vins languedociens, l'échec, sa crainte et ses conséquences ont des vertus indéniables.

${ }^{40}$ E. Siguret, «La manutention mécanique des vins dans les grands chais modernes », La science et la vie, $\mathrm{n}^{\circ} 57$, juin 1921, $\mathrm{p}$ $32-47$. 